\title{
Microscopy of Microbial Gas Vesicles
}

*Correspondence to:

Kim KW,

Tel: +82-54-530-1246

Fax: +82-54-530-1248

E-mail: kiwoo@knu.ac.kr

Received September 5, 2017

Revised September 16, 2017

Accepted September 17, 2017

\author{
Junhyung Park ${ }^{1}$, Ki Woo Kim ${ }^{1,2, *}$ \\ ${ }^{1}$ School of Ecology and Environmental System, Kyungpook National University, Sangju 37224, Korea \\ ${ }^{2}$ Tree Diagnostic Center, Kyungpook National University, Sangju 37224, Korea
}

\begin{abstract}
Gas vesicles are intracellular gas-filled protein-shelled nanocompartments. The structures are spindle or cylinder-shaped, and typically $0.1 \sim 2 \mu \mathrm{m}$ in length and $45 \sim 250 \mathrm{~nm}$ in width. A variety of prokaryotes including photosynthetic bacteria and halophilic archaea form gas vesicles in their cytoplasm. Gas vesicles provide cell buoyancy as flotation devices in aqueous habitats. They are used as nanoscale molecular reporters for ultrasound imaging for biomedical purposes. The structures in halophilic archaea are poorly resolved due to the low signal-to-noise ratio from the high salt concentration in the medium. Such a limitation can be overcome using focused ion beam-thinning or inelastically scattered electrons. As the concentric bodies ( $200 \mathrm{~nm}$ in diameter) in fungi possess gas-filled cores, it is possible that the concept of gas vesicles could be applied to eukaryotic microbes beyond prokaryotes.
\end{abstract}

Key Words: Buoyancy, Gas vesicle, Nanobubble, Protein shell

\section{INTRODUCTION}

Gas vesicles are intracellular gas-filled protein-shelled nanocompartments. Their function is to provide buoyancy which allows aerophilic bacteria to float into oxygenated surface waters (Walsby, 1994). They also enable cyanobacteria to float up toward the light, stratifying in layers below the water surface (Walsby, 1994). The gas vesicles collapse and disappear when subjected to abrupt pressure increase (Ramsay et al., 2011).

Mature gas vesicles are spindle or cylinder-shaped, and typically $0.1 \sim 2 \mu \mathrm{m}$ in length and $45 \sim 250 \mathrm{~nm}$ in width (Pfeifer, 2012) (Fig. 1A and B). They begin as a bicone (biconical structure) and later become the mature forms of gas vesicles (Fig. 1C). There are characteristic 4.6-nm striations or 'ribs' of the 7 8 $\mathrm{kDa}$ gas vesicle protein $\mathrm{A}$ that are perpendicular to the long axis of the gas vesicles. The presence of gas vesicles can reduce the volume of the cytoplasm during the later stage of gas vesicle formation, which might help cells to survive under stress conditions (Pfeifer, 2012) (Fig. 1D). Enclosed by a $2 \mathrm{~nm}$-thick hydrophobic protein shell, gas vesicles exclude water by surface tension at the hydrophobic inner surface, but permit gas from outside to freely diffuse in and out of their barrier (Shapiro et al., 2014; Walsby, 1994) (Fig. 1E). Therefore, the gas within the vesicle is in equilibrium with the gas dissolved in the cytoplasm or medium (Pfeifer, 2012). But they are not considered to store gas as pressurized balloons. Although gas vesicles are intensively studied in aquatic microbes such as the cyanobacterium Anabaena flos-aquae and the halophilic archaeon Halobacterium salinarum, they have been also observed even in a soil microbe and an enterobacterium (Daviso et al., 2013). As microbial gas vesicle-like structures, lipid- or protein-stabilized gas microbubbles have been used as contrast agents for conventional ultrasound imaging (Shapiro et al., 2014). However, the microbubbles are prone to collapse and bubble fragmentation. There is a growing need in ultrasound-modulated stable contrast agents on the nanoscale or molecular reporters. Gas vesicles derived from natural biological structures could be used as nanoscale molecular reporters for ultrasound imaging, based on their hollow interiors, gas permeability, buoyancy, and optical scattering (Shapiro et al., 2014). Due to their nanoscale size, gas vesicles could potentially exit the intravascular space into solid tumors (Cherin et al., 2017). In addition, they could

(a) This is an open-access article distributed under the terms of the Creative Commons Attribution Non-Commercial License (http://creativecommons.org/licenses/by-nc/4.0) which permits unrestricted noncommercial use, distribution, and reproduction in any medium, provided the original work is properly cited.

Copyrights @ 2017 by Korean Society of Microscopy 

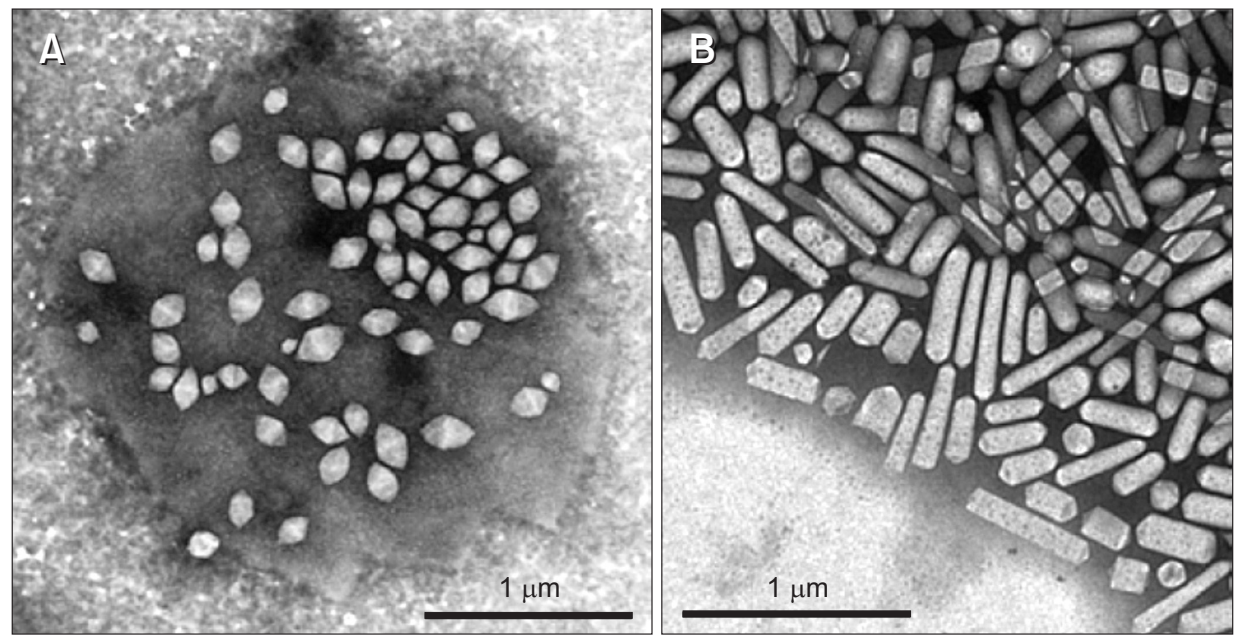

C

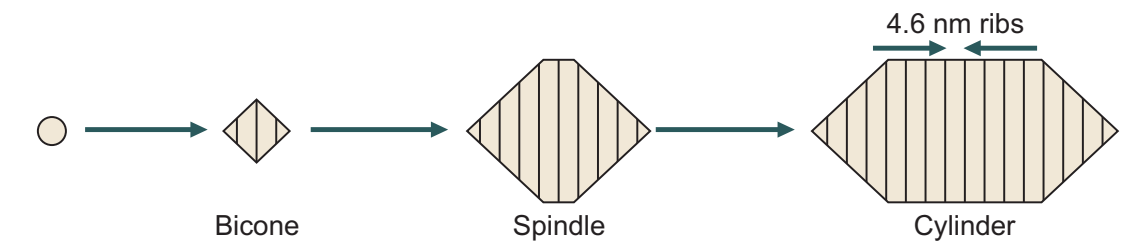

D
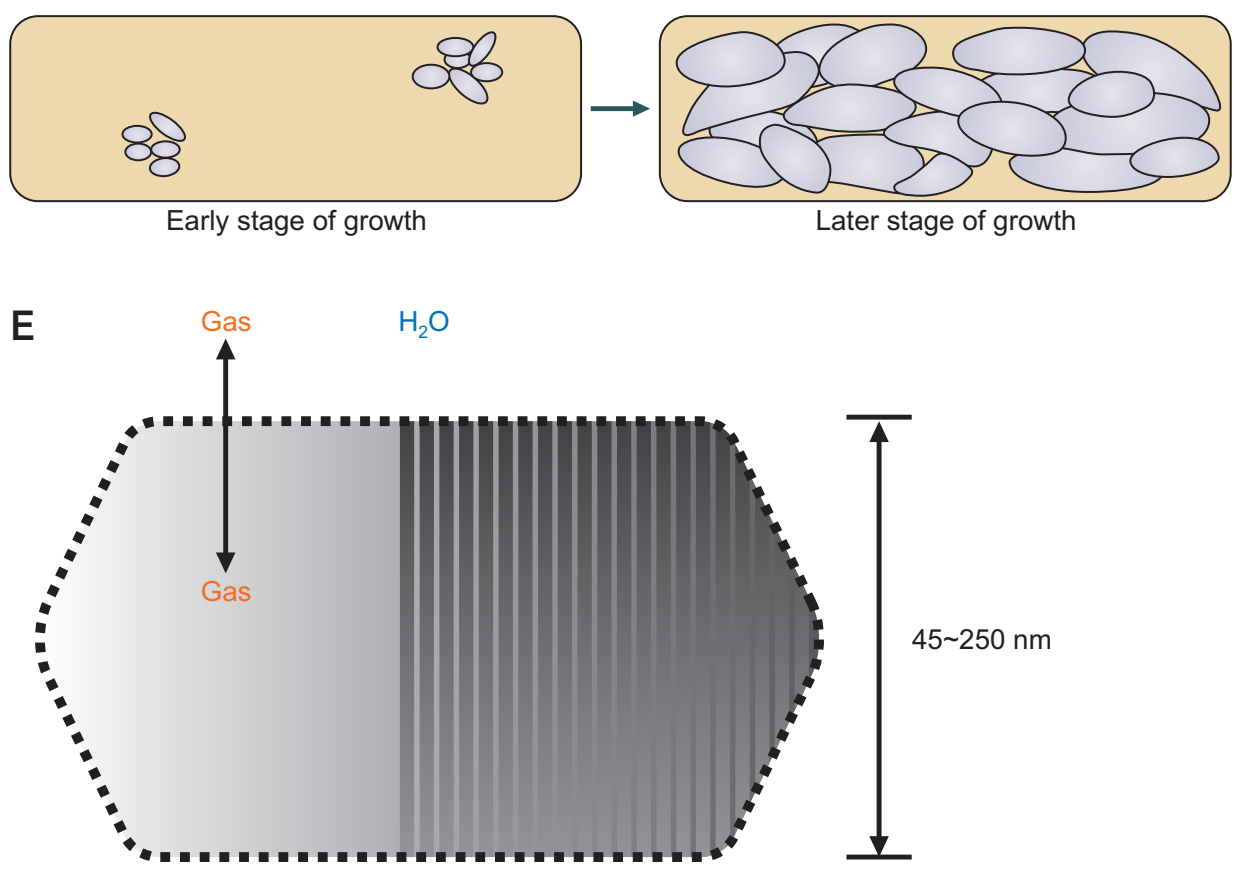

Fig. 1. Gas vesicles. (A) and (B) Transmission electron micrographs of gas vesicles in Halobacterium salinarum. Spindle-shaped gas vesicles in (A). Isolated cylinder-shaped gas vesicles in (B). (C) Morphogenesis of gas vesicles from a bicone to a spindle- or cylindershaped gas vesicle. (D) Groups of gas vesicles. They form clusters during the early stage of gas vesicle formation, and fill the cells later. (E) Detailed diagram of a gas vesicle. A gas nanocompartment enclosed with a gas-permeable shell. (A D) Adapted from Pfeifer (2012) and (E) from Shapiro et al. (2014), with permission from the publisher.

be employed as an antigen presenter for viral or pathogen proteins (Pfeifer, 2015). This review focuses on the structure and microscopy of the most representative gas vesicles in microbes. It could provide a basis for a comprehensive understanding and imaging platforms of microbial gas vesicles for their further exploitation in life sciences and nanotechnology.

\section{PROKARYOTIC GAS VESICLES}

\section{Bacterial Gas Vesicles}

Many bacteria have been known to harbor gas vesicles in their cytoplasm. They include cyanobacteria, anoxygenic photosynthetic bacteria, cold-loving heterotrophic bacteria, spore-forming bacteria, and so on (Pfeifer, 2012). A 
filamentous cyanobacterium A. flos-aquae forms gas vesicles depending on the light intensity, showing more gas vesicles at low light intensities to support an upward movement towards the surface of the aqueous habitat than those at high light intensities (Pfeifer, 2012).

Belonging to the Enterobacteriaceae, a gram-negative enterobacterium Serratia species possesses cylindrical gas vesicles (Fig. 2A). When air was compressed to one-fifth of the original syringe volume, only smaller, diamond-shaped gas vesicles remained (Fig. 2B) (Ramsay et al., 2011). In contrast, gas vesicles are absent in mutant cells deleted for the gas vesicle gene clusters (Fig. 2C). Morphological heterogeneity of gas vesicles occur even in single cells (Fig. 2D). The enterobacterium is known to choose between flagellum-based motility and gas vesicle-based flotation as mutually exclusive taxis modes (Pfeifer, 2012).

\section{Archaeal Gas Vesicles}

Gas vesicles are frequently found in mesophilic archaea living in high-salt environments. The vesicles help the cells to rise from the bottom of the brine, where oxygen concentrations are low (Pfeifer, 2012). Originally isolated as a contaminant from salted fish in 1919, Hbt. salinarum is an extremely halophilic archaeon (Pfeifer, 2015). To harvest light energy, the

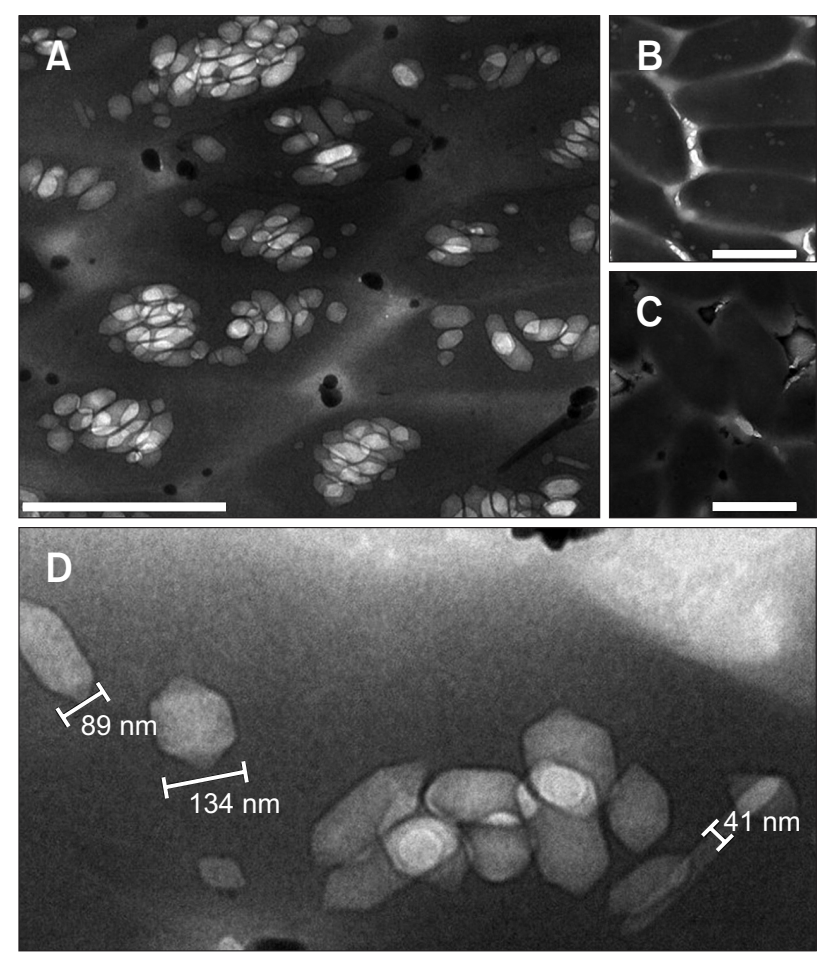

Fig. 2. Transmission electron micrographs of gas vesicles in Serratia species. (A) Wild-type cells. (B) Pressurized wild-type cells. (C) Mutant cells deleted for the gas vesicle gene clusters. (D) Gas vesicles with different widths. Bars $=1 \mu \mathrm{m}$. (A D) Adapted from Ramsay et al. (2011), with permission from the publisher. species uses bacteriorhodopsin that forms crystalline purple patches in the cytoplasmic membrane (Pfeifer, 2015). Gas vesicle-containing cells show pink, opaque colonies on solid media due to the light-interacting properties of gas vesicles; however, cells without gas vesicles form red, transparent colonies (Fig. 3A).

Hbt. salinarum grows optimally in media containing 4.3 $\mathrm{M} \mathrm{NaCl}$ which are far from common isosmotic solutions supplemented with ca. $0.14 \mathrm{M} \mathrm{NaCl}$ for mostly mammalian cells (Bollschweiler et al., 2017). Cryo-transmission electron microscopy of Hbt. salinarum is challenging due to the low signal-to-noise ratio derived from the exceptionally high
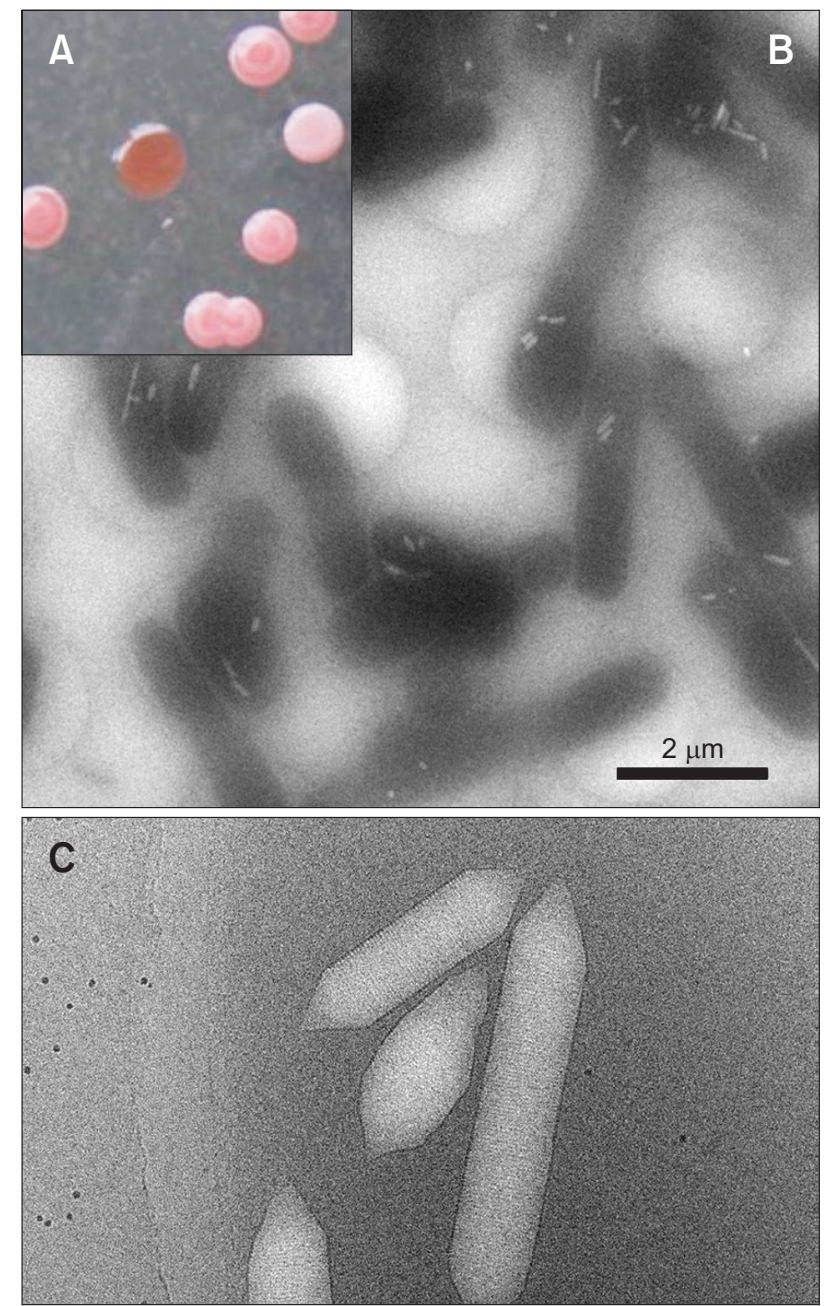

Fig. 3. Gas vesicles in Halobacterium salinarum. (A) Colonies on a solid medium. Pink, opaque colonies from gas vesicle-containing cells; a red, transparent colony from gas vesicle-deficient cells. (B) Cryo-transmission electron micrograph of cells in $3 \mathrm{M} \mathrm{NaCl}$ plus $81 \mathrm{mM} \mathrm{MgSO}_{4}$. The image has the low signal-to-noise ratio due to the high concentration of $\mathrm{NaCl}$. (C) Cryo-transmission electron micrograph of a focused ion beamthinned cell in $3 \mathrm{M} \mathrm{NaCl}$ plus $81 \mathrm{mM} \mathrm{MgSO}_{4}$. The periodicity of the gas vesicle is clearly discerned. (A) Adapted from Pfeifer (2015), (B, C) from Bollschweiler et al. (2017), with permission from the publisher. 
salt concentration in the medium and cytoplasm (Fig. 3B). To overcome this limitation, cryo-focused ion beam (FIB) milling was employed to thin intact vitrified cells, leading to a better image quality with a resolved vesicle ultrastructure (Fig. 3C).

An alternative to the cryo-FIB milling is the application of inelastically scattered electron imaging rather than the most commonly used zero-loss imaging (Kim, 2009). The high salt concentration apparently increases the frequencies of inelastic electron scattering, resulting in the position shift of the highest peak of transmitted electrons. For a higher signal-to-noise ratio in image acquisition, the slit position of an installed energy filter should be shifted toward the low or high-loss region. Such a modification in energy filtering for halophilic archaea can unravel structural details of gas vesicles hidden in poorly resolved micrographs when cryoFIB milling is not available.

\section{PUTATIVE EUKARYOTIC GAS VESICLES}

\section{Fungal Gas Vesicles}

Little information is available on the occurrence of gas vesicles in eukaryotes. However, there are some candidates for putative gas vesicles found in the cytoplasm of eukaryotes. Their proposed structure and functions are similar to those of prokaryotic gas vesicles. Once referred to as ellipsoidal bodies, concentric bodies are found in a variety of fungi (Fig. 4A). They are round in shape and ca. $200 \mathrm{~nm}$ in diameter (Kim \& Kim, 2017). The structures are composed of a gasfilled electron-transparent core and an electron-dense shell with a radiating fibrillar sheath (Fig. 4B). They have been speculated to be involved in cytoplasmic cavitation processes for desiccation tolerance by keeping desiccation-inflicted fungal protoplasts in contact with cell walls (Kim et al.,
2004). It is possible that concentric bodies might function as nanobubbles ( $<1 \mu \mathrm{m}$ in diameter) or gas vesicles in the fungal cytoplasm (Kim \& Kim, 2017). It was conceived by freezeetching results that concentric bodies have a gas-filled center surrounded by proteinaceous material (Honegger, 2007). However, the chemical compositions of concentric bodies were not elucidated thoroughly.

Concentric bodies appear partially similar to gas-filled liposomes synthesized in vitro in morphology (Fig. 5A). The gas-filled liposomes are round in shape and ca. $195 \mathrm{~nm}$ in diameter after negative staining in transmission electron micrographs. In particular, the gas-filled liposomes are characterized by multilayers of different electron densities after gas encapsulation and bubble coalescence. Phospholipids in nanobubble solutions could be assembled to form multilayer gas-filled liposomes (Tian et al., 2015) (Fig. 5B). However, we do not exclude the possibility that a gas-filled core of concentric bodies is enclosed with proteinaceous materials. Further studies will be performed to unravel the structural details of vitrified concentric bodies in fungi using cryo-FIB milling.

\section{CONCLUSIONS}

Gas vesicles have been intensively studied in the domains of bacteria and archaea. The spindle or cylinder-shaped structures are nanocompartments where the gas in the vesicles is in equilibrium with that in the cytoplasm. Apart from buoyancy-associated archetypal gas vesicles in aquatic cyanobacteria and halophilic archaea, mobility-associated gas vesicles regulated by oxygen availability and quorum-sensing mechanism have been characterized in an enterobacterium. These instances imply the prevalent occurrence of gas vesicles in prokaryotes. However, there is a scarcity of studies on the
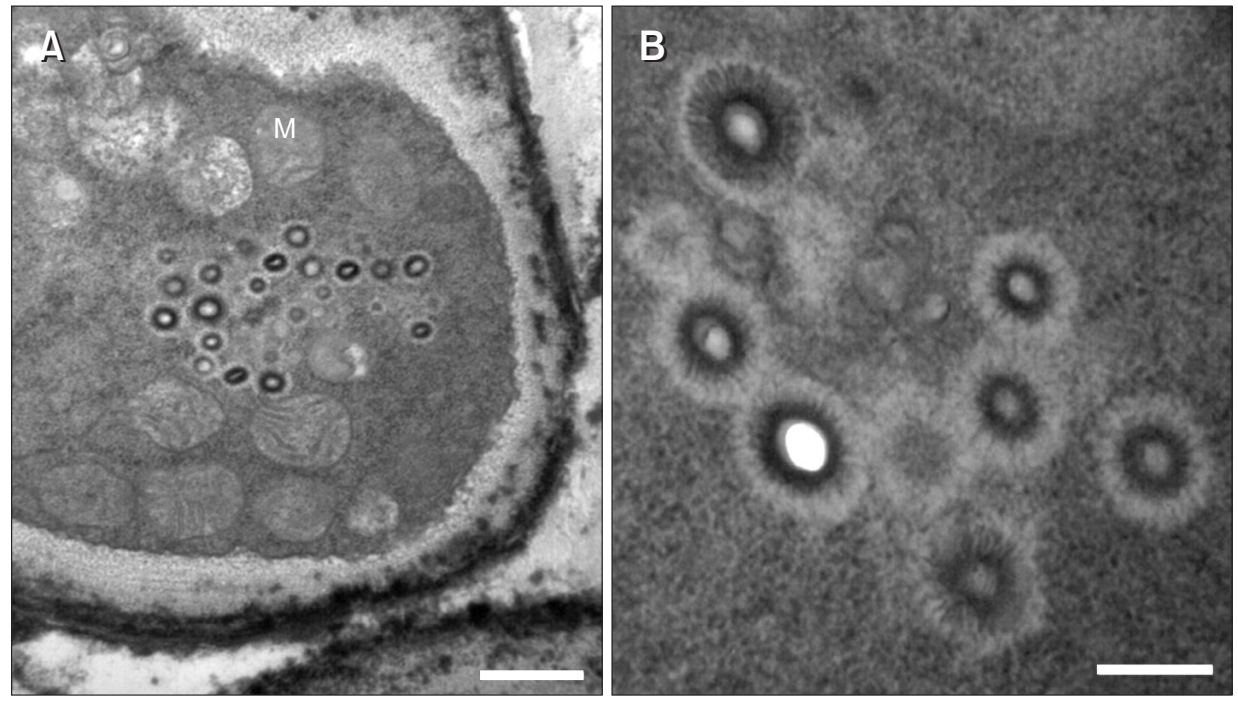

Fig. 4. Transmission electron micrographs of sooty molds on crape myrtle leaves. (A) Cluster of concentric bodies. $\mathrm{M}$, mitochondrium. Bar $=500 \mathrm{~nm}$. (B) Higher magnification of concentric bodies. Bar=200 nm. (A, B) Adapted from Kim \& Kim (2017), with permission from the publisher. 


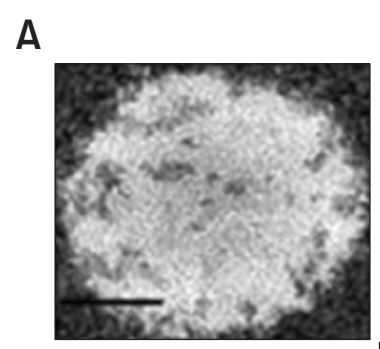

Time

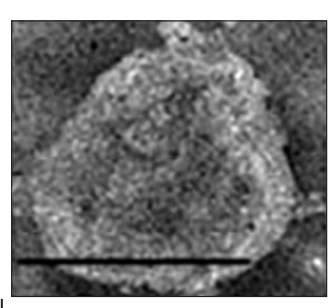

$5 \mathrm{~h}$

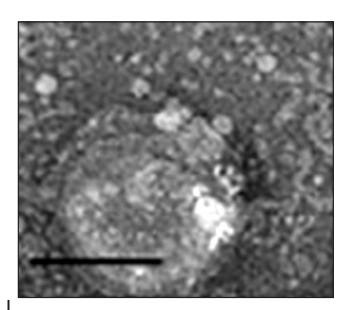

$8 \mathrm{~h}$

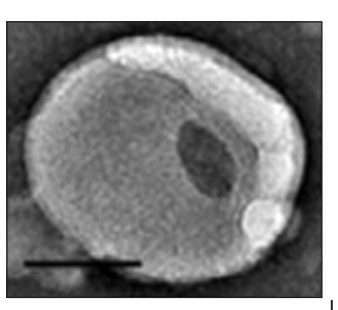

$12 \mathrm{~h}$

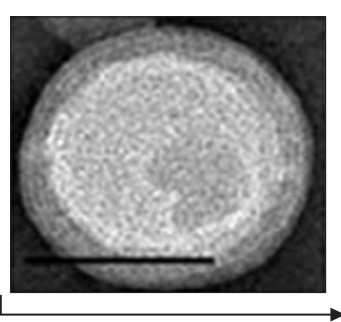

Shaking well

B
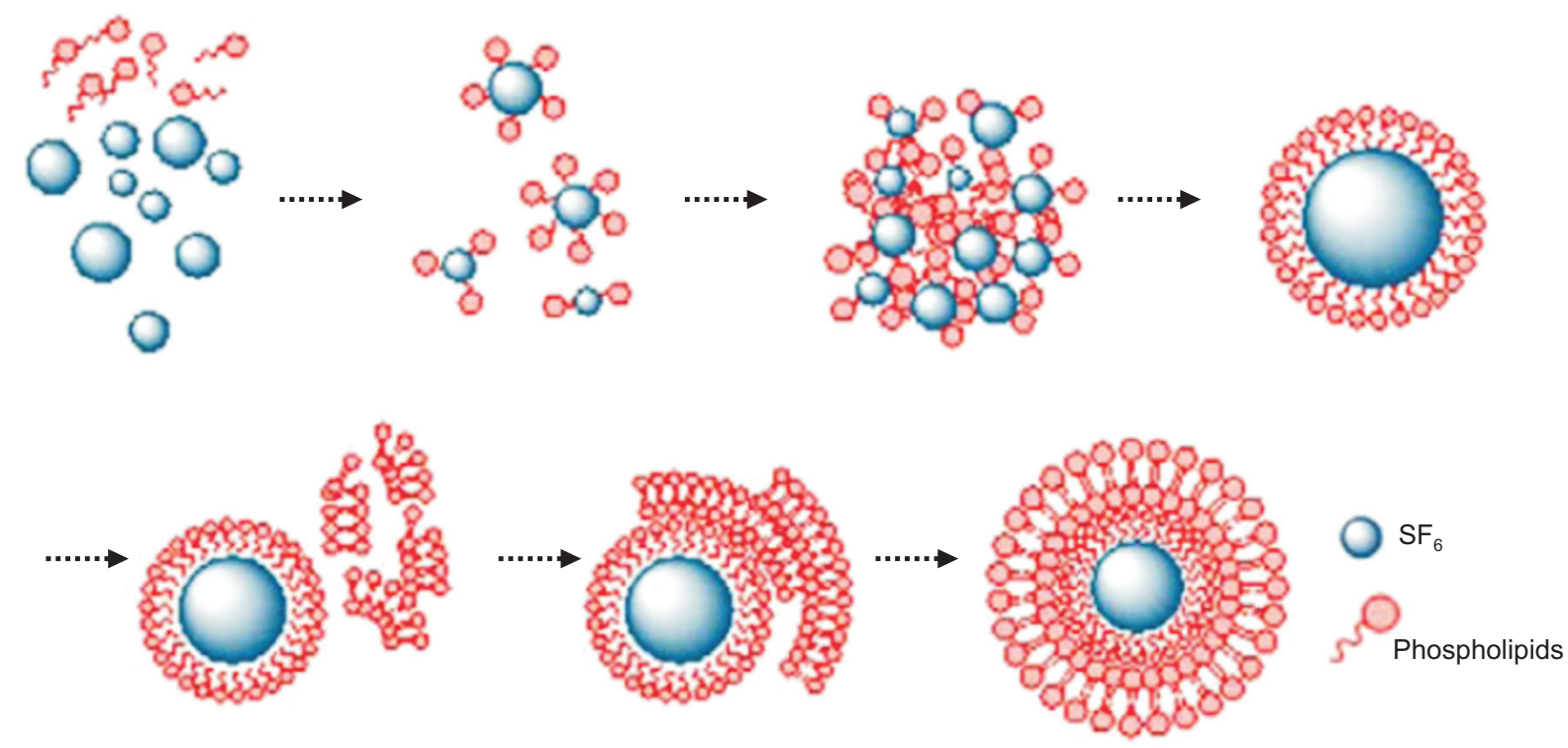

Fig. 5. Gas-filled liposomes. (A) Transmission electron micrographs at different reaction time points. Bars=100 nm. (B) Schematic diagram of the hypothetical formation. Blue=gas core. Red=phospholipids. (A, B) Adapted from Tian et al. (2015), with permission from the publisher.

occurrence of gas vesicles in eukaryotes. Globose structures (ca. $200 \mathrm{~nm}$ in diameter) with gas-filled cores, commonly called concentric bodies, have been reported mostly from lichenized fungi. Considering that the gas-filled core is a requisite for gas vesicles, the concentric bodies could be referred to as putative gas vesicles in eukaryotes. Furthermore, concentric bodies are partially similar to gas-filled liposomes synthesized in vitro, based on their shape, size, and multilayers of different electron densities. To characterize the structural details of microbial gas vesicles, in situ thinning and energyfiltering for enhanced signals of inelastically scattered electrons await further investigations. The occurrence of gas vesicles might not be confined to the domains of bacteria and archaea.

\section{CONFLICT OF INTEREST}

No potential conflict of interest relevant to this article was reported.

\section{ACKNOWLEDGMENTS}

This research was supported by Kyungpook National University Research Fund, 2016.

\section{REFERENCES}

Bollschweiler D, Schaffer M, Lawrence C M, and Engelhardt H (2017) Cryo-electron microscopy of an extremely halophilic microbe: technical aspects. Extremophiles 21, 393-398.
Cherin E, Melis J M, Bourdeau R W, Yin M, Kochmann D M, Foster F S, and Shapiro M G (2017) Acoustic behavior of Halobacterium salinarum gas vesicles in the high-frequency range: experiments and modeling. Ultrasound in Med. \& Biol. 43, 1016-1030. 
Daviso E, Belenky M, Griffin R G, and Herzfeld J (2013) Gas vesicles across kingdoms: a comparative solid-state nuclear magnetic resonance study. J. Mol. Microbiol. Biotechnol. 23, 281-289.

Honegger R (2007) Water relations in lichens. In: Fungi in the Environment, eds. Gadd G M, Watkinson S C and Dyer P, pp. 185200, (Cambridge University Press, Oxford).

Kim J H and Kim K W (2017) Electron microscopic observations of sooty moulds on crape myrtle leaves. For. Path. In press.

Kim K W (2009) Direct whole-mount imaging of fungal spores by energyfiltering transmission electron microscopy. Micron 40, 279-283.

Kim K W, Hyun J W, and Park E W (2004) Cytology of cork layer formation of citrus and limited growth of Elsinoe fawcettii in scab lesions. Eur. J. Plant Pathol. 110, 129-138.

Pfeifer F (2012) Distribution, formation and regulation of gas vesicles. Nat. Rev. Microbiol. 10, 705-715.
Pfeifer F (2015) Haloarchaea and the formation of gas vesicles. Life $\mathbf{5}$, 385-402.

Ramsay J P, Williamson N R, Spring D R, and Salmond G P C (2011) A quorum-sensing molecule acts as a morphogen controlling gas vesicle organelle biogenesis and adaptive flotation in an enterobacterium. Proc. Natl. Acad. Sci. 108, 14932-14937.

Shapiro M G, Goodwill P W, Neogy A, Yin M, Foster F S, Schaffer D V, and Conolly S M (2014) Biogenic gas nanostructures as ultrasonic molecular reporters. Nat. Nanotechnol. 9, 311-316.

Tian J, Yang F, Cui H, Zhou Y, Ruan X, and Gu N (2015) A novel approach to making the gas-filled liposome real: based on the interaction of lipid with free nanobubble within the solution. ACS Appl. Mater. Interfaces 7, 26579-26584.

Walsby A E (1994) Gas vesicles. Microbiol. Rev. 58, 94-144. 